\title{
Localized Subcarinal Adenitis following Endobronchial Ultrasound-Guided Transbronchial Needle Aspiration
}

\author{
Albert Sánchez-Font ${ }^{a, b}$ Luis Álvarez ${ }^{c} \quad$ Gabriela Ledesma ${ }^{a}$ b Víctor Curulla,b \\ a Servei de Pneumologia, Hospital del Mar, Parc de Salut Mar, UAB, CIBERES, ISCIII, and ' IMIM, Hospital del Mar \\ Medical Research Institute, Barcelona, Spain; 'Servei de Pneumologia, Hospital Nostra Senyora de Meritxell, \\ Escaldes-Engordany, Andorra
}

\section{Established Facts}

- Complications associated with endobronchial ultrasound-guided transbronchial needle aspiration (EBUS-TBNA) are rare and include hemorrhage, pneumothorax and infection, mainly mediastinal abscess and mediastinitis.

- We know that germs colonizing the oropharynx may have been dragged along by the echobronchoscope, inoculating the punctured mediastinal lymph node.

\section{Novel Insight}

- We report the first case of localized adenitis as a rare complication of EBUS-TBNA described in the literature.

\section{Key Words}

Endobronchial ultrasound-guided transbronchial needle aspiration · Adenitis · Complications

\begin{abstract}
Endobronchial ultrasound-guided transbronchial needle aspiration (EBUS-TBNA) is a minimally invasive procedure for the diagnosis of mediastinal lymph nodes and masses. Its complications are rare and include hemorrhage, pneumothorax and infections such as mediastinitis. We report the case of a 51-year-old patient who presented with a local-
\end{abstract}

ized subcarinal adenitis after EBUS-TBNA. Germs colonizing the oropharynx may have been dragged along by the echobronchoscope, inoculating the punctured mediastinal lymph node.

(c) 2015 S. Karger AG, Basel

\section{KARGER 125}

E-Mail karger@karger.com www.karger.com/res
2015 S. Karger AG, Basel

0025-7931/15/0904-0329\$39.50/0

\section{Introduction}

Endobronchial ultrasound-guided transbronchial needle aspiration (EBUS-TBNA) is a minimally invasive technique useful in the diagnosis of mediastinal lymph 
Fig. 1. Sections of an axial chest $\mathrm{CT}$ of the mediastinal, window and subcarinal lymph nodes. a Subcarinal lymph node of $24 \mathrm{~mm}$ (short axis) before EBUS. b Subcarinal lymph node of $31 \mathrm{~mm}$ (short axis) with a hypodense center and peripheral uptake, probably related to abscessification, $72 \mathrm{~h}$ after the EBUS with puncture. c Subcarinal lymph node of $19 \mathrm{~mm}$ (short axis) 1 month following the procedure.

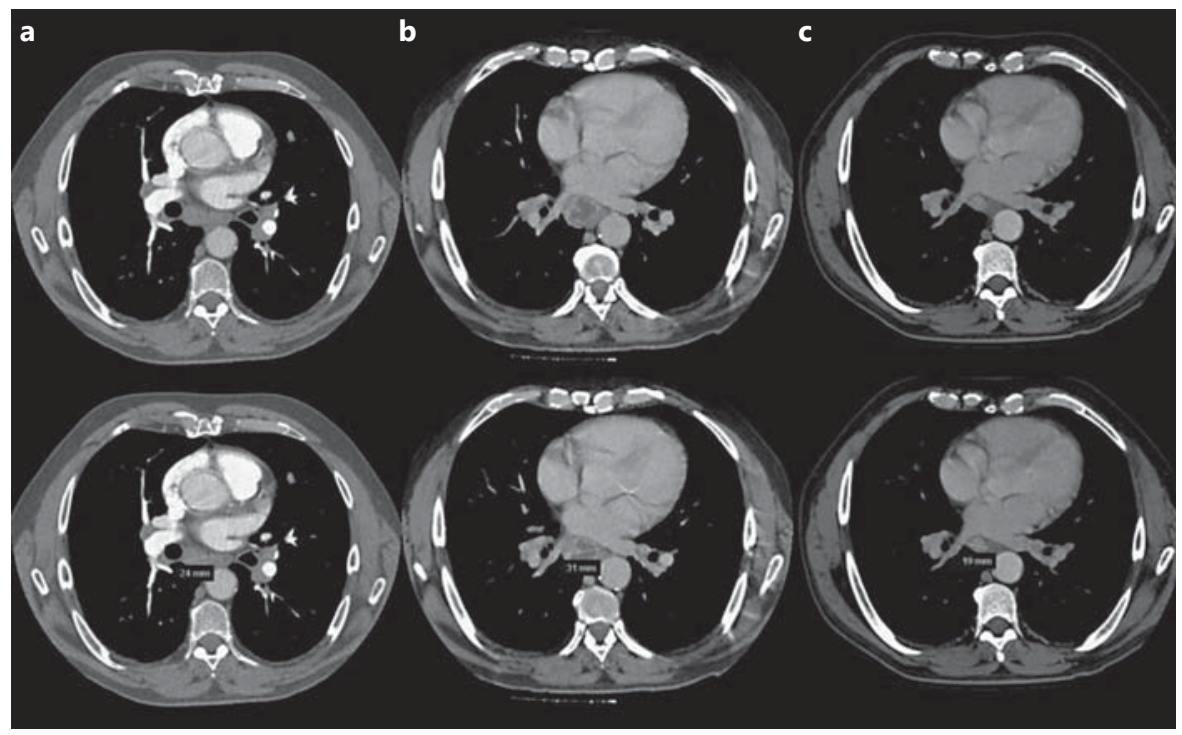

nodes and masses. Complications associated with this procedure are rare and include hemorrhage, pneumothorax and infection, mainly mediastinal abscess. We report the first case of localized adenitis as a rare complication of EBUS-TBNA described in the literature.

\section{Case Report}

We report the case of a 51-year-old man, a former smoker, who worked laying bricks and tarring roads. His medical history was unremarkable, and he had not undergone any previous treatment. A 5-year follow-up chest CT for lung nodules detected multiple bilateral hilar and mediastinal lymph nodes, namely the right paratracheal, prevascular and subcarinal lymph nodes as well as those in the aortopulmonary window (fig. 1a). An EBUS-TBNA was performed in the subcarinal adenopathy, and the echographic images suggested an isoechoic texture without necrosis. The pathology report described the presence of lymphoid cells with 3-dimensional histiocytic aggregates filled with anthracotic pigment and the presence of silica crystals after polarization, suggesting silico-anthracosis. Seventy-two hours following the procedure, the patient developed fever $\left(38.5^{\circ} \mathrm{C}\right)$ and high cervical and retrosternal pain that worsened with swallowing and deep inspiration. Blood tests showed leukocytosis of $12.5 \times 10^{3} / \mathrm{Ul}(80.5 \%$ neutrophils) and a polymerase chain reaction of $41.3 \mathrm{mg} / \mathrm{l}$, which increased to 184.8 $\mathrm{mg} / \mathrm{l}$ the following day. A chest CT showed a slight increase in size of the subcarinal adenopathy, which had a heterogeneous density, hypodense center and peripheral uptake, suggestive of an abscess (fig. 1b). No edema or mediastinal fat trabeculation, signs of acute mediastinitis, were observed. The presence of Gemella morbillorum was detected in two blood cultures. The patient had a favorable clinical, biochemical and radiological outcome and was discharged on amoxicillin/clavulanic acid, $2 \mathrm{~g}$ every $12 \mathrm{~h}$ for 1 month (fig. 1c).

\section{Discussion}

EBUS-TBNA is a safe diagnostic procedure, with few complications. In a meta-analysis including 1,299 patients, complications were found in $0.07 \%$ of the cases [1]. Another systematic review of 14 articles reported no complications [2]. The complications described in the literature have been minor bleeding [3], pneumothorax $[1,4]$, intramural hematoma [5], pneumomediastinum [6], hemopneumomediastinum [5], bacteremia [7] and mediastinitis [8]. Steinfort et al. [7] reported a 7\% incidence of bacteremia following EBUS-TBNA, comparable to that detected after conventional bronchoscopy. Despite a low complication rate, mediastinal infections are considered to be potentially serious, with a mortality of $12-50 \%$, depending on the series [8]. The germs involved are of oropharyngeal origin and include Klebsiella pneumoniae, Actinomyces odontolyticus, Streptococcus mutans, S. pneumoniae and S. viridans $[9,10]$. We were unable to find a report of a localized adenitis secondary to EBUS-TBNA.

In this case, CT (fig. 1b) showed a lymph node with a necrotic core, which was an adenitis that included a necrotic area and an inner abscessification. It is not a mediastinitis because a mediastinitis is an inflammation of the mediastinum with evidence of mediastinal and subcutaneous air. A mediastinal abscess is driven by the evolution of the previously described process with an enclosed collection of liquefied tissue. Therefore, if mediastinitis has occurred recently, we can see it along with a liquid density of the inner area. As the mediastinitis progresses, a 
mediastinal edema with an infiltrative region of soft-tissue attenuation, which obliterates normal mediastinal fat, planes and encases or invades adjacent structures, whereas the liquid will increase in the periphery as the capsule develops. Nevertheless, a mediastinal abscess is never found inside a lymph node, yet outside.

Epstein et al. [11] quantitatively cultured samples by conventional (or blind) TBNA in 7 consecutive patients; all samples showed the growth of polymicrobial aerobic and anaerobic bacteria. They postulated that as the bronchoscope passes through the oropharyngeal region, and subsequently the TBNA needle passes through its interior, they might be contaminated by germs that could be dragged along and inoculate the region during puncture. In this sense, Gemella morbillorum is a Gram-positive, anaerobic bacterium that can colonize the oropharynx and rarely causes disease in humans. It would not be necessary, therefore, for the patient to experience infectious complications. Because of this case, we reviewed the 705 EBUS-TBNAs performed in 504 patients during the past
6 years at our hospital. In 51 cases (7.2\%), signs of bronchial infection were observed during bronchoscopy. However, after the procedure, no infectious complications were detected in any case.

Serious adverse events have been more frequently reported in patients undergoing endoscopic ultrasoundguided fine-needle aspiration (EUS-FNA; 0.3\%) than in patients undergoing EBUS-TBNA (0.05\%). Patients with cystic lesions are bound to have infectious complications because an inoculation of bacteria might path the way to a local uninhibited bacterial growth, as the immune system cannot reach the lesion, and cystic lesions are avascular. In patients with sarcoidosis, however, the low antimicrobial response in the lymph nodes may be the reason for the infectious complications [12].

For all these reasons, we report the first localized adenitis as a rare complication of EBUS-TBNA and, from the experience gained so far, we would postulate that the presence of bronchial infection during EBUS should not preclude performing EBUS-TBNA.

\section{References}

-1 Gu P, Zhao YZ, Jiang LY, et al: Endobronchial ultrasound-guided transbronchial needle aspiration for staging of lung cancer: a systematic review and meta-analysis. Eur J Cancer 2009;45:1389-1396.

-2 Varela-Lema L, Fernández-Villar A, RuanoRavina A: Effectiveness and safety of endobronchial ultrasound-transbronchial needle aspiration: a systematic review. Eur Respir J 2009;33:1156-1164.

$>3$ Vincent B, Huggins JT, Doelken P, et al: Successful real-time endobronchial ultrasoundguided transbronchial needle aspiration of a hiliar lung mass obtained by traversing the pulmonary artery. J Thorac Oncol 2006;1: 362-364.

4 Eapen GA, Shah AM, Lei X, et al: Complications, consequences, and practice patterns of endobronchial ultrasound-guided transbronchial needle aspiration: results of the AQUIRE registry. Chest 2013;143:1044-1053.
5 Botana-Rial M, Nuñez-Delgado M, PallaresSanmartin A, et al: Intramural hematoma of the pulmonary artery and hemopneumomediastinum after endobronchial ultrasoundguided transbronchial needle aspiration. Respiration 2012;83:353-356.

6 Franke KJ, Bruckner C, Szyrach M, et al: The contribution of endobronchial ultrasoundguided forceps biopsy in the diagnostic workup of unexplained mediastinal and hiliar lymphadenopathy. Lung 2012;190:227-232.

7 Steinfort DP, Johnson DF, Irving LB: Incidence of bacteraemia following endobronchial ultrasound-guided transbronchial needle aspiration. Eur Respir J 2010;36:28-32.

8 Asano F, Aoe M, Ohsaki Y, et al: Complications associated with endobronchial ultrasound-guided transbronchial needle aspiration: a nationwide survey by the Japan Society for Respiratory Endoscopy. Respir Res 2013; $14: 50$
-9 Haas AR: Infectious complications from full extension endobronchial ultrasound transbronchial needle aspiration. Eur Respir J 2009; 33:935-938.

10 Steinfort DP, Johnson DF, Irving LB: Infective complications from endobronchial ultrasound-transbronchial needle aspiration. Eur Respir J 2009;34:524-525.

11 Epstein SK, Winslow CJ, Brecher SM, et al: Polymicrobial bacterial pericarditis after transbronchial needle aspiration. Case report with an investigation on the risk of bacterial contamination during fiber optic bronchoscopy. Am Rev Respir Dis 1992;146:523-525.

12 von Bartheld MB, van Breda A, Annema JT: Complication rate of endosonography (endobronchial and endoscopic ultrasound): a systematic review. Respiration 2014;87:343-351. 